\title{
Aggregation of Indistinguishability Fuzzy Relations Revisited
}

\author{
Juan-De-Dios González-Hedström ${ }^{1}$, Juan-José Miñana ${ }^{1,2} \mathbb{D}$ and Oscar Valero ${ }^{1,2, * \mathbb{D}}$ \\ 1 Departament de Ciències Matemàtiques i Informàtica, Universitat de les Illes Balears, \\ 07122 Palma de Mallorca, IB, Spain; j.gonzalez-hedstrom@uib.es (J.-D.-D.G.-H.); jj.minana@uib.es (J.-J.M.) \\ 2 Institut d'Investigació Sanitària Illes Balears (IdISBa), Hospital Universitari Son Espases, \\ 07120 Palma de Mallorca, IB, Spain \\ * Correspondence: o.valero@uib.es
}

Citation: González-Hedström, J.-D.-D.; Miñana, J.-J.; Valero, O. Aggregation of Indistinguishability Fuzzy Relations Revisited. Mathematics 2021, 9, 1441. https://doi.org/10.3390/ math9121441

Academic Editor: Michael Voskoglou

Received: 20 May 2021

Accepted: 15 June 2021

Published: 21 June 2021

Publisher's Note: MDPI stays neutral with regard to jurisdictional claims in published maps and institutional affiliations.

Copyright: (C) 2021 by the authors. Licensee MDPI, Basel, Switzerland. This article is an open access article distributed under the terms and conditions of the Creative Commons Attribution (CC BY) license (https:/ / creativecommons.org/licenses/by/ $4.0 /)$.

\begin{abstract}
Indistinguishability fuzzy relations were introduced with the aim of providing a fuzzy notion of equivalence relation. Many works have explored their relation to metrics, since they can be interpreted as a kind of measure of similarity and this is, in fact, a dual notion to dissimilarity. Moreover, the problem of how to construct new indistinguishability fuzzy relations by means of aggregation has been explored in the literature. In this paper, we provide new characterizations of those functions that allow us to merge a collection of indistinguishability fuzzy relations into a new one in terms of triangular triplets and, in addition, we explore the relationship between such functions and those that aggregate extended pseudo-metrics, which are the natural distances associated to indistinguishability fuzzy relations. Our new results extend some already known characterizations which involve only bounded pseudo-metrics. In addition, we provide a completely new description of those indistinguishability fuzzy relations that separate points, and we show that both differ a lot.
\end{abstract}

Keywords: aggregation; indistinguishability fuzzy relation; extended pseudo-metric; additive generator; continuous Archimedean t-norm

\section{Introduction}

In 1982, the notion of $T$-equivalence (or indistinguishability fuzzy relation with respect to $T$ ), where $T$ is a t-norm, was introduced by E. Trillas in order to provide a fuzzy notion of equivalence relation [1]. Let us recall that a $T$-equivalence on a non-empty set $X$ is a fuzzy set $E: X \times X \rightarrow[0,1]$, which satisfies for each $x, y, z \in X$ the following axioms:

(E1) $E(x, x)=1$;

(E2) $E(x, y)=E(y, x)$;

(E3) $T(E(x, y), E(y, z)) \leq E(x, z)$.

A $T$-equivalence $E$ is said to be a $T$-equality (or indistinguishability fuzzy relation with respect to $T$ that separate points) in those cases in which $E(x, y)=1$ implies $x=y$ ([2]).

Observe that the numerical value $E(x, y)$ provides a degree up to which the element $x$ is indistinguishable from the element $y$. Thus, the greater the $E(x, y)$, the more indistinguishable are $x$ and $y$. In particular, for T-equalities, $E(x, y)=1$ when $x=y$.

Since indistinguishability fuzzy relations were introduced by Trillas, many authors have explored their properties, methods for generating them, and their applications (see, for instance, [2-16]).

From now on we will assume that the reader is familiar with the basics of $t$-norms. For a fuller treatment of the topic, we refer the reader to [17].

The dual relationships between $T$-equivalences and metrics have been deeply explored in the literature, since $T$-equivalences can be interpreted as a kind of measure of similarity and this is, in some sense, a dual notion to dissimilarity which is provided by distances. With the aim of introducing one of the aforementioned relationships, let us recall the 
notion of an extended pseudo-metric. On account of [18], an extended pseudo-metric on a (non-empty) set $X$ is a function $d: X \times X \rightarrow[0, \infty]$ such that for all $x, y, z \in X$ :

(d1) $d(x, x)=0$,

(d2) $d(x, y)=d(y, x)$,

(d3) $d(x, z) \leq d(x, y)+d(y, z)$.

An extended metric $d$ on $X$ is an extended pseudo-metric which satisfies the condition $\left(d 1^{\prime}\right)$ given for all for all $x, y \in X$ as follows: $\left(d 1^{\prime}\right) d(x, y)=0 \Leftrightarrow x=y$.

Following [19], an extended pseudo-metric $d$ on $X$ is called a $b$-bounded pseudometric whenever there exists $b \in \mathbb{R}_{++}$such that $d(x, y) \leq b$ for all $x, y \in X$, where $\mathbb{R}_{++}=\left\{a \in \mathbb{R}_{+}: a>0\right\}$.

Regarding the aforementioned duality, a $T$-equivalence $E_{d, f_{T}}$ can be generated from an extended pseudo-metric $d$ on a non-empty set $X$ and a continuous Archimedean t-norm $T$ as follows (see [20]):

$$
E_{d, f_{T}}(x, y)=f_{T}^{(-1)}(d(x, y)) \text { for all } x, y \in X,
$$

where $f_{T}$ denotes an additive generator for $T$. Notice that the continuity of the $t$-norm cannot be weakened in order to guarantee that $E_{d, f_{T}}$ is a $T$-equivalence, as proved in [21]. The same construction gives a $T$-equality when the extended pseudo-metric is in fact an extended metric [22].

Aggregation theory and aggregation functions play a central role in order to generate methods for constructing new indistinguishability fuzzy relations from older ones. The research activity in aggregation methods has been very intense in the last few years due to potential applications to many problems that arise in a natural way in decision-making. For a deeper discussion of the topic, we refer the reader to [23-26].

Taking this fact into account, a characterization of those functions that allow to aggregate a collection of indistinguishability fuzzy relations into a new one was provided in [27].

With the aim of exposing such a characterization, let us recall, given a collection of $t$-norms $\mathcal{T}=\left\{T_{i}\right\}_{i=1}^{n}$, that a collection of fuzzy binary relations $\left\{E_{i}\right\}_{i=1}^{n}(n \in \mathbb{N})$ is said to be a collection of $\mathcal{T}$-equivalences provided that each $E_{i}$ is a $T_{i}$-equivalence on a non-empty set $X$ for all $i=1, \ldots, n$ (see [3,27]). Moreover, given a t-norm $T$, a function $F$ : $[0,1]^{n} \rightarrow[0,1]$ aggregates $\mathcal{T}$-equivalences into a $T$-equivalence provided that $F\left(E_{1}, \ldots, E_{n}\right)$ is a $T$-equivalence on a non-empty set $X$ when $\left\{E_{i}\right\}_{i=1}^{n}$ is a collection of $\mathcal{T}$-equivalences on $X$. Furthermore, according to [27], a function $G:[0, \infty]^{n} \rightarrow[0, \infty]$ aggregates extended pseudo-metrics into an extended pseudo-metric provided that $G\left(d_{1}, \ldots, d_{n}\right)$ is an extended pseudo-metric on a non-empty set $Y$, whenever $\left\{d_{i}\right\}_{i=1}^{n}$ is a collection of extended pseudometrics on $Y$. In those cases in which the extended pseudo-metrics are bounded, a function $H:\left[0, b_{i}\right]^{n} \rightarrow[0, c]$ is said to aggregate a collection of $\left(b_{i}\right)_{i=1}^{n}$-bounded pseudo-metrics $\left\{d_{i}\right\}_{i=1}^{n}$ on $Y$ if $H\left(d_{1}, \ldots, d_{n}\right)$ is a $c$-bounded pseudo-metric on $Y$ whenever each $d_{i}$ is a $b_{i}$-bounded pseudo-metric on $Y$ for all $i=1, \ldots, n$.

In view of the exposed notions, the aforementioned characterization of indistinguishability fuzzy relations can be stated as follows (see [27]):

Theorem 1. Let $n \in \mathbb{N}$ and let $\mathcal{T}=\left\{T_{i}\right\}_{i=1}^{n}$ be a collection of continuous Archimedean $t$-norms. If $T$ is a continuous Archimedean $t$-norm and $F:[0,1]^{n} \rightarrow[0,1]$ is a function, then the following assertions are equivalent:

(1) F aggregates $\mathcal{T}$-equivalences into a $T$-equivalence.

(2) The function $H: \prod_{i=1}^{n}\left[0, f_{T_{i}}(0)\right]^{n} \rightarrow\left[0, f_{T}(0)\right]$ aggregates every collection $\left\{d_{i}\right\}_{i=1}^{n}$ of $\left(f_{T_{i}}(0)\right)_{i=1}^{n}$-bounded pseudo-metrics into a $f_{T}(0)$-bounded pseudo-metric, where $H=f_{T} \circ$ $F \circ\left(f_{T_{1}}^{-1} \times \ldots \times f_{T_{n}}^{-1}\right)$.

In the light of the preceding result, it must be stressed that $F$ aggregates $\mathcal{T}$-equivalences into a $T$-equivalence, all of them being defined on an arbitrary set $X$. Moreover, the 
function $H$ aggregates every collection of $\left(f_{T_{i}}(0)\right)_{i=1}^{n}$-bounded pseudo-metrics into a $f_{T}(0)$-bounded pseudo-metric, all of them being defined on arbitrary set $Y$. Observe that there is no a priori relationship between $X$ and $Y$. Furthermore, it must be pointed out that the proof of the above result is based on the fact that, given a $T$-equivalence, one can always induce an extended pseudo-metric $d$ by means of $d(x, y)=f_{T}(E(x, y))$ (see [21,22]) and vice versa, given an extended pseudo-metric on $Y$, one can generate a $T$-Equivalence on $Y$ by means of (1).

Notice that the next result is obtained from the preceding one in the particular case in which all continuous Archimedean t-norms are assumed to be strict (see [27]).

Corollary 1. Let $n \in \mathbb{N}$ and let $\mathcal{T}=\left\{T_{i}\right\}_{i=1}^{n}$ be a collection of strict continuous Archimedean $t$-norms. If $T$ is a strict continuous Archimedean $t$-norm and $F:[0,1]^{n} \rightarrow[0,1]$ is a function, then the following assertions are equivalent:

(1) $\quad F$ aggregates $\mathcal{T}$-equivalences into a $T$-equivalence.

(2) The function $H:[0,+\infty]^{n} \rightarrow[0, \infty]$ aggregates every collection $\left\{d_{i}\right\}_{i=1}^{n}$ of extended pseudometrics into an extended pseudo-metric, where $H=f_{T} \circ F \circ\left(f_{T_{1}}^{-1} \times \ldots \times f_{T_{n}}^{-1}\right)$.

It must be stressed that, in the statements of the previous results, the t-norms have been assumed to be continuous and Archimedean in order to warranty that the technique for generating indistinguishability fuzzy relations given by (1) can be applied.

Recently, a new characterization of functions that aggregate indistinguishability fuzzy relations was yielded in terms of triangular triplets with respect to a family of t-norms [28]. The mentioned notion of triangular triplet can be formulated in the following way.

Given a collection of t-norms $\mathcal{T}=\left\{T_{i}\right\}_{i=1}^{n}$, a triplet $(a, b, c) \in[0,1]^{n}$ is said to be a $n$-dimensional $\mathcal{T}$-triangular triplet provided, for all $i=1, \ldots, n$, that

$$
T_{i}\left(a_{i}, b_{i}\right) \leq c_{i}, T_{i}\left(a_{i}, c_{i}\right) \leq b_{i} \text { and } T_{i}\left(b_{i}, c_{i}\right) \leq a_{i}
$$

Observe that the preceding concept retrieves that of a triangular triplet with respect to a t-norm $T$, given in [3], when one considers the collection of t-norms $\mathcal{T}$ such that $T_{i}=T$ for all $i=1, \ldots, n$.

The promised characterization was stated as follows [28].

Theorem 2. Let $n \in \mathbb{N}$ and let $\mathcal{T}=\left\{T_{i}\right\}_{i=1}^{n}$ be a collection of $t$-norms. If $T$ is a $t$-norm and $F$ : $[0,1]^{n} \rightarrow[0,1]$ is a function, then the following assertions are equivalent:

(1) F aggregates $\mathcal{T}$-equivalences into a $T$-equivalence.

(2) F holds the following conditions:

(2.1) $F\left(1_{n}\right)=1$, where $1_{n} \in[0,1]^{n}$ with $1_{n}=(1, \ldots, 1)$.

(2.2) $F$ transforms $n$-dimensional $\mathcal{T}$-triangular triplets into a one-dimensional $T$-triangular triplet.

Let us recall that from Theorem 2, one can retrieve the characterization of functions that aggregate $T$-equivalences into a $T$-equivalence provided in [3]. With this aim, it is sufficient to take the collection of t-norms $\mathcal{T}$ with $T_{i}=T$ for all $i=1, \ldots, n$.

In the particular case of $T$-equalities, the next characterization was also proven in [28].

Theorem 3. Let $n \in \mathbb{N}$ and let $\mathcal{T}=\left\{T_{i}\right\}_{i=1}^{n}$ be a collection of $t$-norms. If $T$ is a $t$-norm and $F$ : $[0,1]^{n} \rightarrow[0,1]$ is a function, then the following assertions are equivalent:

(1) F aggregates $\mathcal{T}$-equalities into a T-equality.

(2) F holds the following conditions:

(2.1) $F\left(1_{n}\right)=1$, where $1_{n} \in[0,1]^{n}$ with $1_{n}=(1, \ldots, 1)$.

(2.2) Let $a \in[0,1]^{n}$. If $F(a)=1$, then there exists $i \in\{1, \ldots, n\}$ such that $a_{i}=1$.

(2.3) If $a, b, c \in\left[0,1\left[^{n}\right.\right.$ such that $(a, b, c)$ is a n-dimensional $\mathcal{T}$-triangular triplet, then, $(F(a), F(b), F(c))$ is a one-dimensional T-triangular triplet. 
Motivated by the exposed facts, the main objectives of this paper are twofold. On the one hand, we provide new information about the description of functions aggregating $\mathcal{T}$-equivalences and their relationship to those that aggregate extended pseudo-metrics. Concretely, we show an equivalence between assertions provided in the statement of Theorems 1 and 2 and the fact that the function $G=f_{T} \circ F \circ\left(f_{T_{1}}^{(-1)} \times \ldots \times f_{T_{n}}^{(-1)}\right)$ aggregates extended pseudo-metrics into a bounded pseudo-metric. On the other hand, we provide a characterization of those functions that aggregate $\mathcal{T}$-equalities into a new one in the spirit of Theorem 1, but taking advantage of Theorem 3. Although it could be expected that such a characterization should be very similar to that given for the case of $\mathcal{T}$-equivalences, it follows that both differ a lot. Thus, roughly speaking, we show that the fact that $F$ aggregates $\mathcal{T}$-equalities into a $T$-equality is equivalent to the fact that the function $G=f_{T} \circ F \circ\left(f_{T_{1}}^{(-1)} \times \ldots \times f_{T_{n}}^{(-1)}\right)$ merges extended metrics into a bounded metric and, in addition, to the fact that the function $G$ transforms $n$-dimensional positive triangular triplets into a one-dimensional positive triangular triplet. Our new results extend the exposed and known characterizations which involve only bounded pseudo-metrics to the extended pseudo-metric framework, which is the most natural when distances associated to indistinguishability fuzzy relations and additive generators are considered. Besides, it must be pointed out that we provide a completely new description of those indistinguishability fuzzy relations that separate points. Notable differences between both characterizations are shown.

\section{Aggregation of $\mathcal{T}$-Equivalences}

In this section, we focus our efforts on obtaining new equivalences with the statements in Theorems 1, 2 and Corollary 1. With this aim, we recall the notion of a triangular triplet following [3].

A triplet $(a, b, c)$, with $s \in[0, \infty]$ and $a, b, c \in[0, s]^{n}(n \in \mathbb{N})$, forms a $n$-dimensional triangular triplet whenever, for all $i=1, \ldots, n$,

$$
a_{i} \leq b_{i}+c_{i}, b_{i} \leq a_{i}+c_{i} \text { and } c_{i} \leq b_{i}+a_{i}
$$

where $x \leq y$ if $y=\infty$ and $x \in[0, \infty]$ or $x, y \in[0, \infty]$ following the usual order of the real line.

In view of the preceding notion, we introduce two results which will play a central role in our subsequent discussion.

Lemma 1. Let $n \in \mathbb{N}$ and $a, b, c \in[0,+\infty]^{n}$. If $(a, b, c)$ is a n-dimensional triangular triplet and $X=\{x, y, z\}$ with different $x, y, z$, then there exists a collection $\left\{d_{i}\right\}_{i=1}^{n}$ of extended pseudo-metrics on $X$ such that $d_{i}(x, y)=a_{i}, d_{i}(x, z)=b_{i}$ and $d_{i}(z, y)=c_{i}$ for all $i=1, \ldots, n$.

Proof. Define, for each $i \in\{1, \ldots, n\}$, the function $d_{i}: X \times X \rightarrow[0,+\infty]$ by $d_{i}(x, y)=$ $d_{i}(y, x)=a_{i}, d_{i}(x, z)=d_{i}(z, x)=b_{i}, d_{i}(z, y)=d_{i}(y, z)=c_{i}$ and $d_{i}(x, x)=d_{i}(y, y)=$ $d_{i}(z, z)=0$. It is not hard to check that $\left\{d_{i}\right\}_{i=1}^{n}$ is a collection of extended metrics on $X$ because $(a, b, c)$ is a $n$-dimensional triangular triplet.

The same arguments given in the proof of Lemma 1 remain valid for the next result in which $n$-dimensional triangular triplets in $[0,+\infty]^{n}$ are considered.

Lemma 2. Let $n \in \mathbb{N}$ and $a, b, c \in[0,+\infty]^{n}$. If $(a, b, c)$ is a n-dimensional triangular triplet and $X=\{x, y, z\}$ with different $x, y, z$, then there exists a collection $\left\{d_{i}\right\}_{i=1}^{n}$ of extended metrics on $X$ such that $d_{i}(x, y)=a_{i}, d_{i}(x, z)=b_{i}$ and $d_{i}(z, y)=c_{i}$ for all $i=1, \ldots, n$.

The next result gives the new aforesaid equivalences. 
Theorem 4. Let $n \in \mathbb{N}$ and let $\mathcal{T}=\left\{T_{i}\right\}_{i=1}^{n}$ be a collection of continuous Archimedean $t$-norms. If $T$ is a continuous Archimedean $t$-norm and $F:[0,1]^{n} \rightarrow[0,1]$ is a function, then the following assertions are equivalent:

(1) F aggregates $\mathcal{T}$-equivalences into a $T$-equivalence.

(2) The function $G:[0,+\infty]^{n} \rightarrow\left[0, f_{T}(0)\right]$ transforms $n$-dimensional triangular triplets in $[0, \infty]^{n}$ into a one-dimensional triangular triplet in $\left[0, f_{T}(0)\right]$ and $G(0, \ldots, 0)=0$, where $G=f_{T} \circ F \circ\left(f_{T_{1}}^{(-1)} \times \ldots \times f_{T_{n}}^{(-1)}\right)$.

(3) The function $G:[0,+\infty]^{n} \rightarrow\left[0, f_{T}(0)\right]$ aggregates every collection $\left\{d_{i}\right\}_{i=1}^{n}$ of extended pseudo-metrics into a $f_{T}(0)$-bounded pseudo-metric, where $G=f_{T} \circ F \circ\left(f_{T_{1}}^{(-1)} \times \ldots \times\right.$ $\left.f_{T_{n}}^{(-1)}\right)$.

(4) The function $H: \prod_{i=1}^{n}\left[0, f_{T_{i}}(0)\right] \rightarrow\left[0, f_{T}(0)\right]$ transforms $n$-dimensional triangular triplets in $\prod_{i=1}^{n}\left[0, f_{T_{i}}(0)\right]$ into a one-dimensional triangular triplet in $\left[0, f_{T}(0)\right]$ and $H(0 \ldots, 0)=0$, where $H=f_{T} \circ F \circ\left(f_{T_{1}}^{-1} \times \ldots \times f_{T_{n}}^{-1}\right)$.

Proof. $(1) \Rightarrow(2)$. By Theorem 2 we have that $F$ transforms $n$-dimensional $\mathcal{T}$-triangular triplets into one-dimensional T-triangular triplets. Now, let $(a, b, c) \in[0,+\infty]^{n}$ be a $n$-dimensional triangular triplet. Then, it is not hard to check that $(d, e, f) \in[0,1]^{n}$ forms a $n$-dimensional $\mathcal{T}$-triangular triplet, where $d=\left(f_{T_{1}}^{(-1)}\left(a_{1}\right), \ldots, f_{T_{n}}^{(-1)}\left(a_{n}\right)\right)$, e $=$ $\left(f_{T_{1}}^{(-1)}\left(b_{1}\right), \ldots, f_{T_{n}}^{(-1)}\left(b_{n}\right)\right)$ and $f=\left(f_{T_{1}}^{(-1)}\left(c_{1}\right), \ldots, f_{T_{n}}^{(-1)}\left(c_{n}\right)\right)$. Thus, $(F(d), F(e), F(f))$ is a one-dimensional $T$-triangular triplet. It follows that

$$
f_{T}(F(u))+f_{T}(F(v)) \geq f_{T} \circ f_{T}^{(-1)}\left(f_{T}(F(u))+f_{T}(F(v))\right)=f_{T}(T(F(u), F(v))) \geq f_{T}(F(w))
$$

for all $u, v, w \in\{d, e, f\}$, whence we conclude that $(G(a), G(b), G(c))$ is a one-dimensional triangular triplet in $\left[0, f_{T}(0)\right]$.

It remains to show that $G(0, \ldots, 0)=0$. First,

$$
\left(f_{T_{1}}^{(-1)} \times \ldots \times f_{T_{n}}^{(-1)}\right)(0, \ldots, 0)=(1, \ldots, 1)=1_{n} .
$$

Besides, again by Theorem 2 , we have that $F\left(1_{n}\right)=1$. Therefore,

$$
G(0, \ldots, 0)=f_{T} \circ F \circ\left(f_{T_{1}}^{(-1)} \times \ldots \times f_{T_{n}}^{(-1)}\right)(0, \ldots, 0)=f_{T}(1)=0 .
$$

$(2) \Rightarrow(3)$. Consider a collection $\left\{d_{i}\right\}_{i=1}^{n}$ of extended pseudo-metrics on a non-empty set $X$. Then, for every $x, y, z \in X$, we have that $(d, e, f) \in[0, \infty]^{n}$ is a $n$-dimensional triangular triplet, where $d=\left(d_{1}(x, y), d_{2}(x, y), \ldots, d_{n}(x, y)\right), e=\left(d_{1}(x, z), d_{2}(x, z), \ldots, d_{n}(x, z)\right)$ and $f=\left(d_{1}(z, y), d_{2}(z, y), \ldots, d_{n}(y, z)\right)$. Then, $(G(d), G(e), G(f))$ is a one-dimensional triplet in $\left[0, f_{T}(0)\right]$, whence we have that

$$
G\left(d_{1}, \ldots, d_{n}\right)(u, v) \leq G\left(d_{1}, \ldots, d_{n}\right)(u, w)+G\left(d_{1}, \ldots, d_{n}\right)(w, v)
$$

for all $u, v, w \in\{x, y, z\}$. It is clear that $G\left(d_{1}, \ldots, d_{n}\right)(x, y)=G\left(d_{1}, \ldots, d_{n}\right)(y, x)$. In order to show that $G\left(d_{1}, \ldots, d_{n}\right)$ is a $f_{T}(0)$-bounded pseudo-metric on $X$, it remains to check that $G\left(d_{1}, \ldots, d_{n}\right)(x, x)=0$ for all $x \in X$. Indeed, let $x \in X$. Then,

$$
G\left(d_{1}, \ldots, d_{n}\right)(x, x)=G\left(d_{1}(x, x), \ldots, d_{n}(x, x)\right)=G(0, \ldots, 0)=0 .
$$

$(3) \Rightarrow(4)$. First we prove that the function $H$ aggregates a collection of $\left(f_{T_{i}}(0)\right)_{i=1}^{n}$ bounded pseudo-metrics on a non-empty set $X$ into a $f_{T}(0)$-bounded pseudo-metric on $X$. Since $\left\{d_{i}\right\}_{i=1}^{n}$ is a collection of extended pseudo-metrics on $X$, the function $G$ aggregates it into a $f_{T}(0)$-bounded pseudo-metric $G\left(d_{1}, \ldots, d_{n}\right)$ on $X$. However, $H\left(d_{1}, \ldots, d_{n}\right)=$ $G\left(d_{1}, \ldots, d_{n}\right)$. Thus, $H\left(d_{1}, \ldots, d_{n}\right)$ is a $f_{T}(0)$-bounded pseudo-metric on $X$. 
Next, consider a $n$-dimensional triangular triplet $(a, b, c)$ in $\prod_{i=1}^{n}\left[0, t_{i}(0)\right]$. Consider $X=\{x, y, z\}$ with different $x, y, z$. By Lemma 1 , there exists a collection of $\left\{d_{i}\right\}_{i=1}^{n}$ of $\left(f_{T_{i}}(0)\right)_{i=1}^{n}$-bounded pseudo-metrics on $X$ such that $d_{i}(x, y)=a_{i}, d_{i}(x, z)=b_{i}$ and $d_{i}(z, y)=c_{i}$ for all $i=1, \ldots, n$. Then, $H\left(d_{1}, \ldots, d_{n}\right)$ is a $f_{T}(0)$-bounded pseudo-metric on $X$. Thus,

$$
H\left(d_{1}, \ldots, d_{n}\right)(u, v) \leq H\left(d_{1}, \ldots, d_{n}\right)(u, w)+H\left(d_{1}, \ldots, d_{n}\right)(w, v)
$$

for all $u, v, w \in X$. It follows that $H(a)=H\left(d_{1}(x, y), \ldots, d_{n}(x, y)\right), H(b)=H\left(d_{1}(x, z), \ldots\right.$, $\left.d_{n}(x, z)\right)$ and $H(c)=H\left(d_{1}(z, y), \ldots, d_{n}(z, y)\right)$. Hence $H(a) \leq H(b)+H(c)$. Similarly, we can show that $H(b) \leq H(a)+H(c)$ and that $H(c) \leq H(a)+H(b)$. Therefore, $(H(a), H(b)$, $H(c))$ is a one-dimensional triangular triplet in $\left[0, f_{T}(0)\right]$.

It remains to prove that $H(0, \ldots, 0)=0$. However, this is evident, since the collection $\left\{d_{i}\right\}_{i=1}^{n}$ under consideration (given by Lemma 1 ) is formed by $\left(f_{T_{i}}(0)\right)_{i=1}^{n}$-bounded pseudometrics on a non-empty set $X$ and, hence, $d_{i}(x, x)=0$ for all $x \in X$. Thus,

$$
H(0, \ldots, 0)=H\left(d_{1}(x, x), \ldots, d_{n}(x, x)\right)=H\left(d_{1}, \ldots, d_{n}\right)(x, x)=0 .
$$

(4) $\Rightarrow(1)$. Consider a collection $\left\{E_{i}\right\}_{i=1}^{n}$ of $\mathcal{T}$-equivalences on a non-empty set $X$. We must prove that $F\left(E_{1}, \ldots, E_{n}\right)$ is a $T$-equivalence.

First we show that $F\left(E_{1}, \ldots, E_{n}\right)(x, x)=1$ for all $x \in X$. The fact that $E_{i}$ is a $T_{i}$-equality on $X$ for every $i$, gives that $E_{i}(x, x)=1$ for all $x \in X$. Assume that $F\left(E_{1}, \ldots, E_{n}\right)(x, x)<1$. Then, $F\left(1_{n}\right)<1$ and

$$
H(0, \ldots, 0)=f_{T} \circ F\left(f_{T_{1}}^{-1}(0), \ldots, f_{T_{n}}^{-1}(0)\right)=f_{T} \circ F\left(1_{n}\right)>f_{T}(1)=0 .
$$

However, this contradicts the fact that $H(0, \ldots, 0)=0$.

Obviously, $F\left(E_{1}, \ldots, E_{n}\right)(x, y)=F\left(E_{1}, \ldots, E_{n}\right)(y, x)$ since $E_{i}(x, y)=E_{i}(y, x)$ for all $x, y \in X$. Thus, it remains to prove that the following inequality is held for each $x, y, z \in X$ :

$$
T\left(F\left(E_{1}, \ldots, E_{n}\right)(x, y), F\left(E_{1}, \ldots, E_{n}\right)(x, z)\right) \leq F\left(E_{1}, \ldots, E_{n}\right)(x, z) .
$$

The next set $f_{T_{i}}\left(E_{i}(x, y)\right)=a_{i}, f_{T_{i}}\left(E_{i}(y, z)\right)=b_{i}$, and $f_{T_{i}}\left(E_{i}(x, z)\right)=c_{i}$. Since

$$
T_{i}\left(E_{i}(x, y), E_{i}(y, z)\right) \leq E_{i}(x, z),
$$

we have that

$$
f_{T_{i}}^{(-1)}\left(a_{i}+b_{i}\right) \leq f_{T_{i}}^{-1}\left(c_{i}\right)
$$

for all $i=1, \ldots, n$. It follows that

$$
f_{T_{i}} \circ f_{T_{i}}^{(-1)}\left(a_{i}+b_{i}\right) \geq c_{i}
$$

for all $i=1, \ldots, n$. Since

$$
a_{i}+b_{i} \geq f_{T_{i}} \circ f_{T_{i}}^{(-1)}\left(a_{i}+b_{i}\right),
$$

we deduce that $a_{i}+b_{i} \geq c_{i}$ for all $i=1, \ldots, n$. Similar arguments allow us to show that $a_{i}+c_{i} \geq b_{i}$ and that $c_{i}+b_{i} \geq a_{i}$ for all $i=1, \ldots, n$. Consequently, we have that $(c, a, b)$ is a $n$-dimensional triangular triplet in $\prod_{i=1}^{n}\left[0, f_{T_{i}}(0)\right]$, where $c=\left(c_{i}\right)_{i=1}^{n}, a=\left(a_{i}\right)_{i=1}^{n}$ and $b=\left(b_{i}\right)_{i=1}^{n}$. Thus, $(H(c), H(a), H(b))$ is a one-dimensional triangular triplet in $\left[0, f_{T}(0)\right]$. Thus, $H(c) \leq H(a)+H(b)$. Then,

$$
\begin{aligned}
& f_{T} \circ F \circ\left(f_{T_{1}}^{-1}\left(c_{1}\right) \times \ldots \times f_{T_{n}}^{-1}\left(c_{n}\right)\right) \leq \\
& f_{T} \circ F \circ\left(f_{T_{1}}^{-1}\left(a_{1}\right) \times \ldots \times f_{T_{n}}^{-1}\left(a_{n}\right)\right)+f_{T} \circ F \circ\left(f_{T_{1}}^{-1}\left(b_{1}\right) \times \ldots \times f_{T_{n}}^{-1}\left(b_{n}\right)\right) .
\end{aligned}
$$


Applying $f_{T}^{(-1)}$ to both hands of the preceding inequality immediately gives that

$$
T\left(F\left(E_{1}, \ldots, E_{n}\right)(x, y), F\left(E_{1}, \ldots, E_{n}\right)(x, z)\right) \leq F\left(E_{1}, \ldots, E_{n}\right)(x, z) .
$$

Consequently, we conclude that $F\left(E_{1}, \ldots, E_{n}\right)$ is a $T$-equivalence on $X$.

It must be pointed out that if the collection of t-norms $\mathcal{T}$ is reduced to the single continuous Archimedean t-norm $T$, then equivalence $(1) \Leftrightarrow(4)$ in Theorem 4 retrieves the characterization yielded in [3]. Moreover, the combination of Theorem 1 with the aforementioned equivalence improves the characterization provided in [29], where all bounded pseudo-metrics belonging to the collection to be aggregated and the bounded pseudo-metric obtained via the aggregation share the same constant of boundedness.

From Theorem 4, we can derive the following one, which improves Corollary 1, if all continuous Archimedean t-norms are assumed to be strict.

Theorem 5. Let $n \in \mathbb{N}$ and let $\mathcal{T}=\left\{T_{i}\right\}_{i=1}^{n}$ be a collection of strict continuous Archimedean $t$-norms. If $T$ is a strict continuous Archimedean $t$-norm and $F:[0,1]^{n} \rightarrow[0,1]$ is a function, then the following assertions are equivalent:

(1) F aggregates $\mathcal{T}$-equivalences into a T-equivalence.

(2) The function $G:[0,+\infty]^{n} \rightarrow[0,+\infty]$ transforms $n$-dimensional triangular triplets into a one-dimensional triangular triplets in $[0,+\infty]$ and $G(0, \ldots, 0)=0$, where $G=t \circ F \circ$ $\left(f_{T_{1}}^{-1} \times \ldots \times f_{T_{n}}^{-1}\right)$.

(3) The function $G:[0,+\infty]^{n} \rightarrow[0,+\infty]$ aggregates every collection $\left(d_{i}\right)_{i=1}^{n}$ of extended pseudo-metrics into an extended pseudo-metric, where $G=t \circ F \circ\left(f_{T_{1}}^{-1} \times \ldots \times f_{T_{n}}^{-1}\right)$.

\section{Aggregation of $\mathcal{T}$-Equalities}

This section is devoted to deal with the characterization of the functions that allow us to aggregate $\mathcal{T}$-equalities making use of Theorem 3 .

Theorem 6. Let $n \in \mathbb{N}$ and let $\mathcal{T}=\left\{T_{i}\right\}_{i=1}^{n}$ be a collection of continuous Archimedean $t$-norms. If $T$ is a continuous Archimedean $t$-norm and $F:[0,1]^{n} \rightarrow[0,1]$ is a function, then the following assertions are equivalent:

(1) F aggregates $\mathcal{T}$-equalities into a $T$-equality.

(2) The function $G:[0,+\infty]^{n} \rightarrow\left[0, f_{T}(0)\right]$, where $G=f_{T} \circ F \circ\left(f_{T_{1}}^{(-1)} \times \ldots \times f_{T_{n}}^{(-1)}\right)$, fulfills the following conditions:

(2.1) $G(0, \ldots, 0)=0$;

(2.2) Let $a \in[0,+\infty]^{n}$. If $G(a)=0$, then there exists $i \in\{1, \ldots, n\}$ such that $a_{i}=0$;

(2.3) G transforms $n$-dimensional triangular triplets in $] 0,+\infty]^{n}$ into a one-dimensional triangular triplet in $\left.] 0, f_{T}(0)\right]$.

(3) The function G: $[0,+\infty]^{n} \rightarrow\left[0, f_{T}(0)\right]$ aggregates every collection $\left\{d_{i}\right\}_{i=1}^{n}$ of extended metrics into a $f_{T}(0)$-bounded metric, where $G=f_{T} \circ F \circ\left(f_{T_{1}}^{(-1)} \times \ldots \times f_{T_{n}}^{(-1)}\right)$.

(4) The function $H: \prod_{i=1}^{n}\left[0, f_{T_{i}}(0)\right] \rightarrow\left[0, f_{T}(0)\right]$ aggregates every collection $\left\{d_{i}\right\}_{i=1}^{n}$ of $\left(f_{T_{i}}(0)\right)_{i=1^{-}}^{n}$ bounded metrics into a $f_{T}(0)$-metric, where $H=f_{T} \circ F \circ\left(f_{T_{1}}^{-1} \times \ldots \times f_{T_{n}}^{-1}\right)$.

(5) The function $H: \prod_{i=1}^{n}\left[0, f_{T_{i}}(0)\right] \rightarrow\left[0, f_{T}(0)\right]$, where $H=f_{T} \circ F \circ\left(f_{T_{1}}^{-1} \times \ldots \times f_{T_{n}}^{-1}\right)$, fulfills the following conditions:

(5.1) $H(0, \ldots, 0)=0$;

(5.2) Let $a \in \prod_{i=1}^{n}\left[0, f_{T_{i}}(0)\right]$. If $H(a)=0$, then there exists $i \in\{1, \ldots, n\}$ such that $a_{i}=0$;

(5.3) $\quad H$ transforms $n$-dimensional triangular triplets in $\left.\left.\prod_{i=1}^{n}\right] 0, f_{T_{i}}(0)\right]$ into a one-dimensional triangular triplet in $\left.] 0, f_{T}(0)\right]$; 
Proof. (1) $\Rightarrow(2)$. By Theorem 3, we have that $F$ transforms $n$-dimensional $\mathcal{T}$-triangular triplets in $\left[0,1\left[{ }^{n}\right.\right.$ into one-dimensional $T$-triangular triplets and, in addition, $F\left(1_{n}\right)=1$. The same arguments to those given in Theorem 4 show that $G(0, \ldots, 0)=0$ from the fact that $F\left(1_{n}\right)=1$.

Now, assume that $a \in[0,+\infty]^{n}$ with $G(a)=0$. Then,

$$
f_{T} \circ F\left(f_{T_{1}}^{(-1)}\left(a_{1}\right), \ldots, f_{T_{n}}^{(-1)}\left(a_{n}\right)\right)=0 .
$$

It follows that

$$
F\left(f_{T_{1}}^{(-1)}\left(a_{1}\right), \ldots, f_{T_{n}}^{(-1)}\left(a_{n}\right)\right)=1 .
$$

Thus, there exists $i \in\{1, \ldots, n\}$ such that $f_{T_{i}}^{(-1)}\left(a_{i}\right)=1$, whence we obtain that $a_{i}=0$.

Next, consider a $n$-dimensional triangular triplet $(a, b, c)$ in $] 0,+\infty]^{n}$. Then, $u_{i} \leq$ $v_{i}+w_{i}$ for all $i=1, \ldots, n$ and for all $u, v, w \in\{a, b, c\}$. Thus,

$$
f_{T_{i}}^{(-1)}\left(u_{i}\right) \geq f_{T_{i}}^{(-1)}\left(v_{i}+w_{i}\right) \geq T_{i}\left(f_{T_{i}}^{(-1)}\left(v_{i}\right), f_{T_{i}}^{(-1)}\left(w_{i}\right)\right)
$$

for all $u, v, w \in\{a, b, c\}$, whence we deduce that $\left(f_{T_{i}}^{(-1)}\left(a_{i}\right), f_{T_{i}}^{(-1)}\left(b_{i}\right), f_{T_{i}}^{(-1)}\left(c_{i}\right)\right)$ is a $T_{i^{-}}$ triangular triplet. Thus, $(d, e, f) \in\left[0,1\left[{ }^{n}\right.\right.$ is a $n$-dimensional $\mathcal{T}$-triangular triplet in $[0,1[$ with $d=\left(f_{T_{1}}^{(-1)}\left(a_{1}\right), \ldots, f_{T_{n}}^{(-1)}\left(a_{n}\right)\right), e=\left(f_{T_{1}}^{(-1)}\left(b_{1}\right), \ldots, f_{T_{n}}^{(-1)}\left(b_{n}\right)\right)$ and $f=\left(f_{T_{1}}^{(-1)}\left(c_{1}\right), \ldots\right.$, $\left.f_{T_{n}}^{(-1)}\left(c_{n}\right)\right)$. Then, $(F(d), F(e), F(f))$ is a one-dimensional $T$-triangular triplet. Hence, we have that

$$
T(F(u), F(w)) \leq F(v)
$$

for all $u, v, w \in\{d, e, f\}$, whence we deduce that

$$
f_{T}^{(-1)}\left(f_{T} \circ F(u)+f_{T} \circ F(w)\right)=T(F(u), F(w)) \leq F(v)
$$

for all $u, v, w \in\{d, e, f\}$. Thus, we obtain

$$
G(u)+G(w)=f_{T} \circ F(u)+f_{T} \circ F(w) \geq f_{T} \circ\left(f_{T}^{(-1)}\left(f_{T} \circ F(u)+f_{T} \circ F(w)\right)\right) \geq f_{T} \circ F(v)=G(v)
$$

for all $u, v, w \in\{a, b, c\}$. Therefore, $(G(a), G(b), G(c))$ is a one-dimensional triangular triplet in $\left.] 0, f_{T}(0)\right]$. Observe that $G(u)>0$ for all $u \in\{a, b, c\}$, since otherwise we have that there exists $i \in\{1, \ldots, n\}$ such that $u_{i}=0$, which contradicts the fact that $\left.\left.a, b, c \in\right] 0,+\infty\right]^{n}$.

(2) $\Rightarrow(3)$. Consider a collection $\left\{d_{i}\right\}_{i=1}^{n}$ of extended metrics on a non-empty set $X$. We will see that $G\left(d_{1}, \ldots, d_{n}\right)$ is a metric on $X$.

First we show that $G\left(d_{1}, \ldots, d_{n}\right)(x, y)=0 \Leftrightarrow x=y$. On the one hand, if $x=y$, then $d_{i}(x, x)=0$ for all $i=1, \ldots, n$. Thus,

$$
G\left(d_{1}, \ldots, d_{n}\right)(x, x)=G\left(d_{1}(x, x), \ldots, d_{n}(x, x)\right)=G(0, \ldots, 0)=0 .
$$

Now assume we have that $G\left(d_{1}, \ldots, d_{n}\right)(x, y)=0$ for any $x, y \in X$. Then,

$$
G\left(d_{1}(x, y), \ldots, d_{n}(x, y)\right)=0 .
$$

Thus, there exists $i \in\{1, \ldots, n\}$ such that $d_{i}(x, y)=0$. Hence, $x=y$.

Obviously, $G\left(d_{1}, \ldots, d_{n}\right)(x, y)=G\left(d_{1}, \ldots, d_{n}\right)(y, x)$ for each $x, y \in X$.

Finally, we prove that the triangle inequality

$$
G\left(d_{1}, \ldots, d_{n}\right)(x, z) \leq G\left(d_{1}, \ldots, d_{n}\right)(x, y)+G\left(d_{1}, \ldots, d_{n}\right)(y, z)
$$

is held for each $x, y, z \in X$. Without loss of generality, we can assume that the cardinality of $X$ is at least three, that is, that there exists $x, y, z \in X$ with different $x, y, z$. Otherwise, the triangle inequality is clear. 
Define $a=\left(d_{1}(x, z), d_{2}(x, z), \ldots, d_{n}(x, z)\right), b=\left(d_{1}(x, y), d_{2}(x, y), \ldots, d_{n}(x, y)\right)$ and $c=$ $\left(d_{1}(y, z), d_{2}(y, z), \ldots, d_{n}(y, z)\right)$. By our assumptions, $(a, b, c) \in[0,+\infty]^{n}$ is a $n$-dimensional triangular triplet. This gives that $(G(a), G(b), G(c))$ is a one-dimensional triangular triplet in ] $\left.0, f_{T}(0)\right]$, which follows that

$$
G\left(d_{1}, \ldots, d_{n}\right)(x, z) \leq G\left(d_{1}, \ldots, d_{n}\right)(x, y)+G\left(d_{1}, \ldots, d_{n}\right)(y, z)
$$

$(3) \Rightarrow(4)$. It is obvious.

$(4) \Rightarrow(5)$. The same reasoning to that applied in the proof of $(3) \Rightarrow(4)$ in Theorem 4 remains valid here, to show that $H(0, \ldots, 0)=0$.

Next, we prove that if there exists $a \in \prod_{i=1}^{n}\left[0, f_{T_{i}}(0)\right]$ such that $H(a)=0$, then there exists $i \in\{1, \ldots, n\}$ such that $a_{i}=0$. For the purposes of contradiction, assume that $\left.\left.a \in \prod_{i=1}^{n}\right] 0, f_{T_{i}}(0)\right]$ with $H(a)=0$. Consider a non-empty set $X=\{x, y\}$ such that $x \neq y$. Define the function $d_{i}: X \times X \rightarrow\left[0, f_{T_{i}}(0)\right]$ by $d_{i}(x, y)=d_{i}(y, x)=a_{i}$ and $d_{i}(x, x)=d_{i}(y, y)=0$ for all $i=1, \ldots, n$. Then, $\left\{d_{i}\right\}_{i=1}^{n}$ is a collection of $\left(f_{T_{i}}(0)\right)_{i=1}^{n}$ bounded metrics on $X$. Thus, $H\left(d_{1}, \ldots, d_{n}\right)$ is a $f_{T}(0)$-bounded metric on $X$. Since $H(a)=0$, we have that

$$
H\left(d_{1}, \ldots, d_{n}\right)(x, y)=H\left(d_{1}(x, y), \ldots, d_{n}(x, y)\right)=H(a)=0
$$

which is impossible because $x \neq y$. Then, if $H(a)=0$, we deduce that there exists $i \in\{1, \ldots, n\}$ such that $a_{i}=0$.

We need only consider a $n$-dimensional triangular triplet $\left.\left.(a, b, c) \in \prod_{i=1}^{n}\right] 0, f_{T_{i}}(0)\right]$. Set $X=\{x, y, z\}$ with different $x, y, z$. By Lemma 2 we have guaranteed the existence of a collection $\left\{d_{i}\right\}_{i=1}^{n}$ of $\left(f_{T_{i}}(0)\right)_{i=1}^{n}$-bounded metrics on $X$ with $d_{i}(x, y)=a_{i}, d_{i}(x, z)=b_{i}$ and $d_{i}(z, y)=c_{i}$ for all $i=1, \ldots, n$. Then, $H\left(d_{1}, \ldots, d_{n}\right)$ is a $f_{T}(0)$-bounded metric on $X$, whence we deduce that

$$
H\left(d_{1}, \ldots, d_{n}\right)(u, v) \leq H\left(d_{1}, \ldots, d_{n}\right)(u, w)+H\left(d_{1}, \ldots, d_{n}\right)(w, v)
$$

for all $u, v, w \in\{x, y, z\}$. From these inequalities, we obtain that $(H(a), H(b), H(c))$ is a one-dimensional triangular triplet in $\left.] 0, f_{T}(0)\right]$.

$(5) \Rightarrow(1)$. First of all, we show that $F\left(1_{n}\right)=1$. Since

$$
H(0, \ldots, 0)=f_{T} \circ F\left(f_{T_{1}}^{(-1)}(0), \ldots, f_{T_{n}}^{(-1)}(0)\right)=f_{T} \circ F(1, \ldots, 1)
$$

and $H(0, \ldots, 0)=0$, we immediately deduce that $F(1, \ldots, 1)=1$.

Now, assume that $a \in[0,1]^{n}$ with $F(a)=1$. Then,

$$
H\left(b_{1}, \ldots, b_{n}\right)=f_{T} \circ F\left(f_{T_{1}}^{-1}\left(b_{1}\right), \ldots, f_{T_{n}}^{-1}\left(b_{n}\right)\right)
$$

with $b_{i}=f_{T}\left(a_{i}\right)$ for all $i=1, \ldots, n$. The fact that

$$
F\left(f_{T_{1}}^{-1}\left(b_{1}\right), \ldots, f_{T_{n}}^{-1}\left(b_{n}\right)\right)=F(a)=1
$$

gives that $H\left(b_{1}, \ldots, b_{n}\right)=0$. Thus, there exists $i \in\{1, \ldots, n\}$ such that $b_{i}=0$. It follows that $a_{i}=f_{T_{i}}^{-1}(0)=1$.

Finally, let $a, b, c \in[0,1[n$ such that $(a, b, c)$ is a $n$-dimensional $\mathcal{T}$-triangular triplet. Then, $(e, f, g)$ is a $n$-dimensional triangular triplet in $\left.\left.\prod_{i=1}^{n}\right] 0, f_{T_{i}}\right]$, where $e=\left(f_{T_{1}}\left(a_{1}\right), \ldots\right.$, $\left.f_{T_{n}}\left(a_{n}\right)\right), f=\left(f_{T_{1}}\left(b_{1}\right), \ldots, f_{T_{n}}\left(b_{n}\right)\right)$ and $g=\left(f_{T_{1}}\left(c_{1}\right), \ldots, f_{T_{n}}\left(c_{n}\right)\right)$. Indeed, we have that $T_{i}\left(u_{i}, v_{i}\right) \leq w_{i}$ for all $i=1, \ldots, n$ and for all $u, v, w \in\{a, b, c\}$. Hence, we obtain that

$$
f_{T_{i}}^{(-1)}\left(f_{T_{i}}\left(u_{i}\right)+f_{T}\left(v_{i}\right)\right) \leq w_{i}
$$


for all $i=1, \ldots, n$ and for all $u, v, w \in\{a, b, c\}$, whence we deduce that

$$
f_{T_{i}} \circ f_{T_{i}}^{(-1)}\left(f_{T_{i}}\left(u_{i}\right)+f_{T}\left(v_{i}\right)\right) \geq f_{T_{i}}\left(w_{i}\right)
$$

for all $i=1, \ldots, n$ and for all $u, v, w \in\{a, b, c\}$. Since

$$
f_{T_{i}}\left(u_{i}\right)+f_{T}\left(v_{i}\right) \geq f_{T_{i}} \circ f_{T_{i}}^{(-1)}\left(f_{T_{i}}\left(u_{i}\right)+f_{T}\left(v_{i}\right)\right),
$$

we deduce that

$$
f_{T_{i}}\left(u_{i}\right)+f_{T}\left(v_{i}\right) \geq f_{T_{i}}\left(w_{i}\right)
$$

for all $i=1, \ldots, n$ and for all $u, v, w \in\{a, b, c\}$. Consequently, $(H(e), H(f), H(g))$ is a one-dimensional triangular triplet in $\left.] 0, f_{T}(0)\right]$. Thus,

$$
f_{T} \circ F\left(u_{1}, \ldots, u_{n}\right) \leq f_{T} \circ F\left(v_{1}, \ldots, v_{n}\right)+f_{T} \circ F\left(w_{1}, \ldots, w_{n}\right)
$$

for all $u, v, w \in\{a, b, c\}$, since $H(e)=f_{T} \circ F\left(a_{1}, \ldots, a_{n}\right), H(f)=f_{T} \circ F\left(b_{1}, \ldots, b_{n}\right)$ and $H(g)=f_{T} \circ F\left(c_{1}, \ldots, c_{n}\right)$. Thus, we get that

$$
f_{T}^{-1} \circ f_{T} \circ F\left(u_{1}, \ldots, u_{n}\right) \geq f_{T}^{-1}\left(f_{T} \circ F\left(v_{1}, \ldots, v_{n}\right)+\circ f_{T} \circ F\left(w_{1}, \ldots, w_{n}\right)\right)
$$

for all $u, v, w \in\{a, b, c\}$. Thus, we have that

$$
F(u)=F\left(u_{1}, \ldots, u_{n}\right) \geq f_{T}^{-1}\left(f_{T} \circ F\left(v_{1}, \ldots, v_{n}\right)+f_{T} \circ F\left(w_{1}, \ldots, w_{n}\right)\right)=T(F(v), F(w))
$$

for all $u, v, w \in\{a, b, c\}$. Therefore, $(F(a), F(b), F(c))$ is a one-dimensional $T$-triangular triplet. Now, the fact that $F$ aggregates $\mathcal{T}$-equalities into a $T$-equality follows by Theorem 3 .

In the particular case in which all continuous Archimedean t-norms are assumed to be strict, then, from Theorem 6, we can deduce the next result.

Corollary 2. Let $n \in \mathbb{N}$ and let $\mathcal{T}=\left\{T_{i}\right\}_{i=1}^{n}$ be a collection of strict continuous Archimedean $t$-norms. If $T$ is a strict continuous Archimedean t-norm and $F:[0,1]^{n} \rightarrow[0,1]$ is a function, then the following assertions are equivalent:

(1) F aggregates $\mathcal{T}$-equalities into a $T$-equality.

(2) The function $G$ : $[0,+\infty]^{n} \rightarrow[0,+\infty]$, where $G=f_{T} \circ F \circ\left(f_{T_{1}}^{-1} \times \ldots \times f_{T_{n}}^{-1}\right)$, fulfills the following conditions:

(2.1) $G(0, \ldots, 0)=0$;

(2.2) Let $a \in[0,+\infty]^{n}$. If $G(a)=0$, then there exists $i \in\{1, \ldots, n\}$ such that $a_{i}=0$;

(2.3) $G$ transforms $n$-dimensional positive triangular triplets in $[0,+\infty]^{n}$ into a onedimensional positive triangular triplet in $[0,+\infty]$.

(3) The function $G:[0,+\infty]^{n} \rightarrow[0,+\infty]$ aggregates every collection $\left\{d_{i}\right\}_{i=1}^{n}$ of extended metrics into an extended metric, where $G=f_{T} \circ F \circ\left(f_{T_{1}}^{-1} \times \ldots \times f_{T_{n}}^{-1}\right)$.

\section{Conclusions}

In 1982, the notion of indistinguishability fuzzy relations was introduced by E. Trillas in order to provide a fuzzy notion of equivalence relations. In many problems coming from decision-making, new indistinguishability fuzzy relations are induced by means of merging a collection of other indistinguishability fuzzy relations. Motivated by this fact, a characterization of those functions that aggregate indistinguishability fuzzy relations into a new one was given, on the one hand, in terms of functions that aggregate bounded pseudometrics in [27] and, on the other hand, in terms of functions that transform triangular triplets with respect to a t-norm in [3].

In this paper, we have again addressed the aggregation problem of indistinguishability fuzzy relations. Concretely, we have provided a new characterization of those functions that 
merge a collection of such fuzzy relations into a new one based on triangular triplets and a specific function induced by additive generators of the involved t-norms that aggregate extended pseudo-metrics into bounded pseudo-metrics. Finally, a characterization of those functions that aggregate indistinguishability fuzzy relations that separate points have also been given. Notable differences between both characterizations have been shown.

The problem of describing - in terms of aggregation of generalized distances and triangular triplets- those functions that aggregate more general notions of indistinguishability fuzzy relations, like relaxed indistinguishability fuzzy relations or fuzzy preorders, remains open. Thus, we will address it as further work.

Author Contributions: Funding acquisition, O.V.; Investigation, J.-D.-D.G.-H., J.-J.M. and O.V.; Writing-original draft, J.-D.-D.G.-H., J.-J.M. and O.V.; Writing-review and editing, J.-J.M. and O.V. All authors have read and agreed to the published version of the manuscript.

Funding: This research was funded by FEDER/Ministerio de Ciencia, Innovación y UniversidadesAgencia Estatal de Investigación/ _ Proyecto PGC2018-095709-B-C21, Programa Operatiu FEDER 2014-2020 de les Illes Balears, by project PROCOE/4/2017 (Direcció General d'Innovació i Recerca, Govern de les Illes Balears) and from projects ROBINS and BUGWRIGHT2. These two latest projects have received funding from the European Union's Horizon 2020 research and innovation programme under grant agreements No. 779776 and No. 871260, respectively. This publication reflects only the authors views and the European Union is not liable for any use that may be made of the information contained therein.

Institutional Review Board Statement: Not applicable.

Informed Consent Statement: Not applicable.

Data Availability Statement: Not applicable.

Conflicts of Interest: The authors declare no conflict of interest. The funders had no role in the design of the study; in the collection, analyses, or interpretation of data; in the writing of the manuscript, or in the decision to publish the results.

\section{References}

1. Trillas, E. Assaig sobre les relacions d'indistingibilitat. In Proceedings Primer Congrés Català de Lògica Matemàtica; Barcelona Institut d'Estudis Catalans: Barcelona, Spain, 1982; pp. 51-59.

2. Recasens, J. Indistinguishability Operators: Modelling Fuzzy Equalities and Fuzzy Equivalence Relations; Springer: Berlin, Germany, 2010.

3. Mayor, M.; Recasens, J. Preserving T-transitivity. In Artificial Intelligence Research and Development; Nebot, À., Binefa, X., López de Mántaras, R., Eds.; IOS Press: Amsterdam, The Netherlands, 2016; pp. 79-87.

4. Calvo, T.; Fuster-Parra, P. Aggregation of partial $\mathcal{T}$-indistinguishability operators and partial pseudo-metrics. Fuzzy Sets Syst. 2019, 403, 119-138.

5. Miñana, J.-J.; Valero, O. On indistinguishability operators, fuzzy metrics and modular metrics. Axioms 2017, 6, 34.

6. Höhle, U. Fuzzy equalities and indistinguishability. In Proceedings of EUFIT'93; Eurogress Aachen: Aachen, Germany, 1993; Volume 1, pp. 358-363.

7. Höhle, U. Many valued equalities and their representation. In Logical, Algebraic, Analytic and Probabilistic Aspects of Triangular Norms; Klement, E.P., Mesiar, R., Eds.; Elsevier: Amsterdam, The Netherlands, 2005; pp. 301-320.

8. Petry, F. E.; Bosc, P. Fuzzy Databases: Principles and Applications; Kluwer Academic Publishers: Boston, MA, USA 1996.

9. Ovchinnikov, S. Representation of transitive fuzzy relations. In Aspects of Vagueness; Skala, H.J., Termini, S., Trillas, E., Eds., Springer: Berlin/Heidelberg, Germany, 1984; pp. 105-118.

10. Boixader, D.; Recasens, J. On the relationship between fuzzy subgroups and indistinguishability operators. Fuzzy Sets Syst. 2019, $373,149-163$.

11. Calvo, T.; Recasens, J. On the representation of local indistinguishability operators. Fuzzy Sets Syst. 2021, 410, 90-108.

12. Recasens, J. On the Relationship between Positive Definite Matrices and t-norms. Fuzzy Sets Syst. 2021 , in press.

13. Bejines, C.; Chasco, M.J.; Elorza, J.; Recasens, J. Preserving fuzzy subgroups and indistinguishability operators. Fuzzy Sets Syst. 2019, 373, 164-179.

14. Bejines, C.; Ardanza, S.; Chasco, M.J.; Elorza, J. Aggregation of indistinguishability operators. Fuzzy Sets Syst. 2021, in press.

15. Pedraza, T.; Rodríguez-López, J.; Valero, O. Aggregation of fuzzy quasi-metrics. Inform. Sci. 2021, in press.

16. Saminger, S.; Mesiar, R.; Bodenhofer, U. Domination of aggregation operators and preservation of transitivity. Int. J. Uncertain. Fuzziness-Knowl.-Based Syst. 2002, 10, 11-35.

17. Klement, E.P.; Mesiar, R.; Pap, E. Triangular Norms; Kluwer: Dordrecht, The Netherlands, 2000. [CrossRef] 
18. Deza, M.M.; Deza, E. Encyclopedia of Distances; Springer: Heidelberg, Germany, 2009. [CrossRef]

19. Copson, E.T. Metric Spaces; Cambridge University Press: Cambridge, UK, 1968.

20. Valverde, L. On the structure of F-indistinguishability operators. Fuzzy Set. Syst. 1985, 17, 313-328.

21. De Baets, B.; Mesiar, R. Pseudo-metrics and T-equivalences. Fuzzy. Math. 1997, 5, 471-481.

22. De Baets, B.; Mesiar, R. Metrics and T-equalities. J. Math. Anal. Appl. 1997 , 267, 531-547.

23. Beliakov, G.; Bustince, H.; Calvo, T. A Practical Guide to Averaging Functions, Studies in Fuzziness and Soft Computing; Springer: Berlin/Heidelberg, Germany, 2016.

24. Grabisch, M.; Marichal, J.-L.; Mesiar, R.; Pap, E. Aggregation functions, Encyclopedia of Mathematics and its Applications; Cambridge University Press: Cambridge, UK, 2009; Volume 127.

25. Drewniak, J.; Dudziak, U. Aggregation in classes of fuzzy relations. Stud. Math. 2006, 5, 33-43.

26. Drewniak, J.; Dudziak, U. Preservation of properties of fuzzy relations during aggregation processes. Kybernetik 2007, 43, 115-132.

27. Pradera, P.; Trillas, E.; Castiñeira, E. On the aggregation of some classes of fuzzy relations. In Technologies for Constructing Intelligent Systems 2. Tools; Bouchon-Meunier, B., Gutiérrez-Ríos, J., Magdalena, L., Yager, R.R., Eds.; Springer: Heidelberg, Germany, 2002; pp. 125-147. [CrossRef]

28. Calvo Sánchez, T.; Fuster-Parra, P.; Valero, O. The aggregation of transitive fuzzy relations revisited. Fuzzy Sets Syst. 2021, in press. [CrossRef]

29. Pradera, P.; Trillas, E. A note on pseudo-metrics aggregation, Int. J. Gen. Syst. 2002 , 31, 41-51. [CrossRef] 